\title{
Predictors of Chat Usage in an App-Based Health Coaching Platform: Implications for Treatment Program Planning
}

\author{
Woo, K.M., Lee, J.K.
}

Naluri Hidup Sdn. Bhd., Kuala Lumpur, Malaysia

\begin{abstract}
Introduction: Digital healthcare with smartphone application is a promising approach to bridge the gap between hospital and community care by facilitating preventive health behavior and chronic illness management. Such technology allows healthcare professionals to integrate online coaching and consultation as part of the treatment process.
\end{abstract}

Objectives: This study seeks to understand the factors predicting users' usage of the chat function in an app-based health coaching platform.

Methods: A total of 394 employees from an oil and gas company participated in health screening as part of their human resource initiatives for employee wellness. Demographic variables, biometrics, psychometrics, and other variables were collected as part of the screening. Employees with existing chronic illness (i.e. diabetes and cardiovascular disease) or the risk of developing one, as indicated by their body mass index ([BMI] $>27$ ), blood pressure $(>140 / 90)$, HbAlc (> 5.9), cholesterol (> 5.2), and depression/ anxiety/ stress levels (moderate to severe), are enrolled into a 4-month online coaching program.

Pearson correlation was performed between all the variables, together with users' total chat count in four months. Subsequently, significant variables were entered into a multiple linear regression model using the forward method, with total message count as the dependent variable.

Results: The analysis included 278 sample who have been offered the coaching program. Significant zero-order correlation was found between total chat count, waistline, family history of high cholesterol, self-identified areas of improvement, ongoing medical follow up, and cholesterol level. At the multivariate level $\left(\mathrm{R}^{2}=.119, \mathrm{~F}[1,198]=26.62, \mathrm{p}<.001\right)$, only total number of self- identified areas of improvement was found to be significant $(\beta=.34, p<.01)$.

Conclusion: This study shows that the number of self-identified areas of improvement is an important factor that predicts user chat activity in online health coaching.

Keywords: Digital healthcare, smartphone application, online coaching 